\title{
Hypothyroidism - An Underestimated Complication After Hemithyroidectomy: A Preliminary Study Report
}

\author{
Neeraj Aggarwal, ${ }^{1}$ Sirshak Dutta, ${ }^{1}$ Tanaya Panja, ${ }^{1}$ Mainak Dutta, ${ }^{1}$ Sanjoy Kumar Ghosh, ${ }^{1}$ Ramanuj Sinha ${ }^{1}$
}

\begin{abstract}
Introduction
Hypothyroidism is a significant but an under-appreciated complication of hemithyroidectomy. The focus of this study was to assess the incidence of hypothyroidism occurring after hemithyroidectomy, identify the risk factors and to suggest a possible follow-up guideline for diagnosis.

Materials and Methods

An institution based preliminary study was conducted in the Department of Otorhinolaryngology, in a tertiary care hospital, from August 2014 to December 2015 on 25 patients. The patients were regularly followed up in 1 st month, 3rd month, 6th month, 9 th month and 1 year period with the thyroid function test.

Results

Incidence of hypothyroidism occurring after hemithyroidectomy including transient and permanent cases is $32 \%$. Increased age of presentation, longer duration of thyroid swelling, pre-operative serum TSH level $>2.5 \mathrm{mIU} / \mathrm{ml}$, lymphocytic infiltration in histopathology and right sided hemithyroidectomy were found to be the possible risk factors.

Conclusion

It is indicated from this preliminary report that there should be a serial thyroid function monitoring in every post-operative hemithyroidectomy patient, and special attention should be given to the risk group

Kevwords

Hypothyroidism, Hemithyroidectomy
\end{abstract}

$\underline{\text { ABSTRACT }}$

$\mathrm{H}$ emithyroidectomy refers to removal of one lobe of thyroid along with the isthmus and pyramidal lobe, if present. Although hypothyroidism after hemithyroidectomy is an underrecognized complication, some recent studies showed that it does exist. The incidence of hypothyroidism after hemithyroidectomy as reported in various literature is between 0 to 43 percent. ${ }^{1}$ Hypothyroidism can be either subclinical or overt. Normal serum TSH level is $0.43-4.25 \mathrm{mIU} / \mathrm{ml}^{2}{ }^{2}$ Subclinical hypothyroidism means elevation of TSH beyond the upper limit of the reference range, with normal free T4 level, without any symptom. ${ }^{3,4}$ Overt hypothyroidism means elevation of serum TSH level above the reference range and decrease in free $\mathrm{T} 4$ level below the reference range along with clinical symptoms. ${ }^{4,5}$ So serum TSH is considered to be the most sensitive and earliest detectable parameter to diagnose post-operative hypothyroidism. ${ }^{2}$ There are certain other risk factors which can be assessed preoperatively and indicate the increased chance of postoperative hypothyroidism. ${ }^{3}$ Therefore, identification of these risk factors at an early stage can prevent long term complication of hypothyroidism.

But the incidence of post-operative hypothyroidism after hemithyroidectomy remains unpredictable ${ }^{6}$ and a long term follow-up is required to analyze these patients. Differences in opinion still persist regarding the causes, risk factors, and management of hypothyroidism following hemithyroidectomy due to dearth of studies. At present, there is no universal protocol to assess thyroid function after hemithyroidectomy.
1 - Department of ENT, Medical College, Kolkata
Corresponding author:
Dr Tanaya Panja
email: tanypnj@gmail.com 
So, the purpose of the present study was to assess the incidence, to find out the causes and risk factors and to work-up a possible follow-up protocol for early diagnosis of hypothyroidism after hemithyroidectomy.

\section{Materials and Methods}

An institution based preliminary study was conducted in the Department of Otorhinolaryngology, in a tertiary care hospital, from August 2014 to December 2015 on 25 patients. A preliminary report had been prepared from the study up to December 2015. The patients who were operated between August 2014 and December 2014 were followed up for a period of 1 year.

All the patients who underwent hemithyroidectomy for benign pathology irrespective of age, sex, socio-economic and demographic characteristics were included in the study whereas patients with deranged thyroid profile pre-operatively and/or taking medications for that, or any other medication which interferes with thyroid function status and all thyroid malignancies were excluded from the study. After performing hemithyroidectomy, the samples were sent for histopathology and the patients who required completion thyroidectomy after hemithyroidectomy were also excluded from the study. The patients were regularly followed up in 1st month, 3rd month, 6th month, 9th month and 1 year period with the thyroid function tests and status of thyroid function was assessed post-operatively.

\section{Results}

The study was conducted on 25 patients who were followed up periodically for one year. The preliminary results shown below are based on this one year followup data.

At the end of periodic follow-up after 1 year, we encountered 5 patients who developed permanent hypothyroidism, including overt and sub-clinical cases. The patient showing sub-clinical or overt hypothyroidism in any of the follow-ups and becoming euthyroid later on and maintaining the euthyroid status for next two or more follow-ups without any supplementation therapy were termed as 'transient case' in our study. The patients having either normal thyroid function status or subclinical hypothyroidism were followed up in regular intervals without imposing any supplementation therapy. Levothyroxine supplementation therapy was started in overt cases only, and they were followed up after 3 months. If they attained euthyroid status, levothyroxine supplementation was stopped. The follow-up of each hypothyroid patient has been individualized and their progression of hypothyroidism in the follow-up period is shown in Fig. 1.

At the end of 1 year follow-up, the overall incidence of permanent hypothyroidism (that includes subclinical and overt cases) was $20 \%$ (5 out of 25 patients). Amongst the permanent cases, 2 subclinical (40\%) and 3 overtly hypothyroid $(60 \%)$ cases were found. In the study we also found 3 transient cases out of total study population (12\%). The total incidence including temporary and permanent hypothyroidism in the present study was $32 \%$.

On distribution of the patients in different age groups (Fig. 2), most cases of post-operative hypothyroidism were observed in the age group of 46-55 years (2 out of 3 cases, $66.7 \%$ ) and 36-45 years (4 out of 10 cases, $40 \%$ ). Patients in the age group of 26-35 years ( 2 out of 9 cases, $18.9 \%$ ) showed comparatively less incidence of post-operative hypothyroidism, while in the age group of 16-25 years no case of hypothyroidism was reported.

It had also been observed that the patients having pre-operative mean TSH level $>2.5 \mathrm{mIU} / \mathrm{ml}$ had more incidence of post-operative hypothyroidism (6 out of 13 patients, $46.2 \%$ ) as compared to patients who had pre-operative mean TSH level $<2.5 \mathrm{mIU} / \mathrm{ml}$ ( 2 out of 12 patients, 16.7\%), as shown in Fig. 3. The mean TSH level pre-operatively in all the hypothyroid patients (including transient and permanent, total 8) was $2.87 \mathrm{mIU} / \mathrm{ml}$ and in the euthyroid patients it was 1.65 $\mathrm{mIU} / \mathrm{ml}$.

The incidence of hypothyroidism was more in patients who were having thyroid swelling for more than 10 years ( 7 out of 17 patients, $41.2 \%$ ). No case of post-operative hypothyroidism was found in patients having thyroid swelling less than 5 years.(Fig. 4) This 

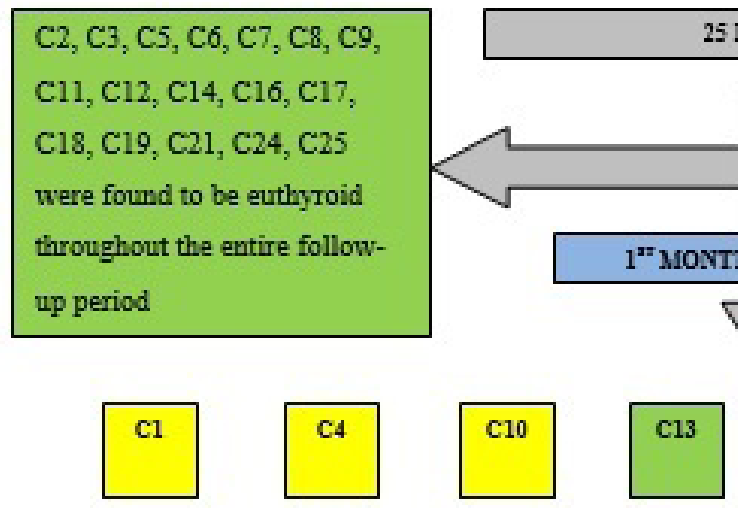

25 Patients
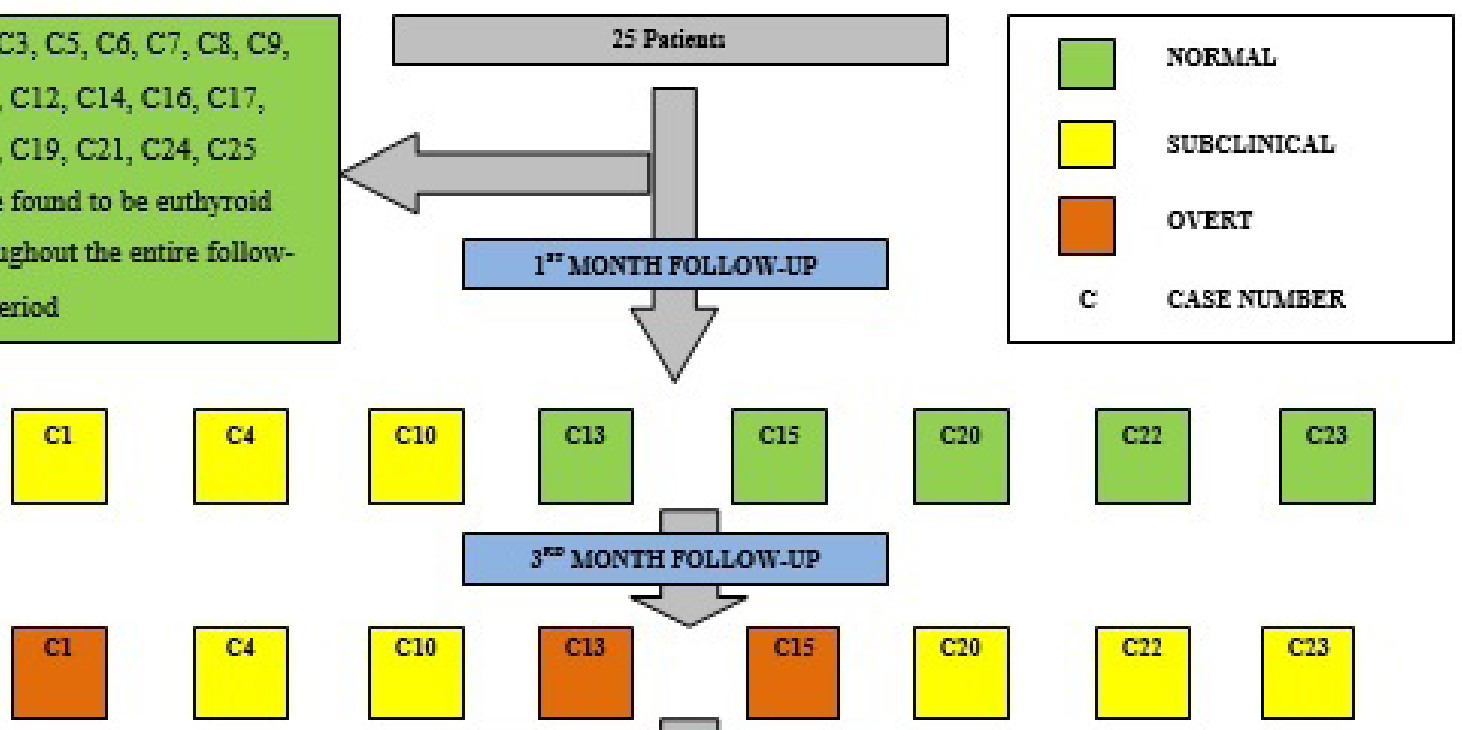

$6^{\text {TI }}$ MONTH FOLLOW-UP
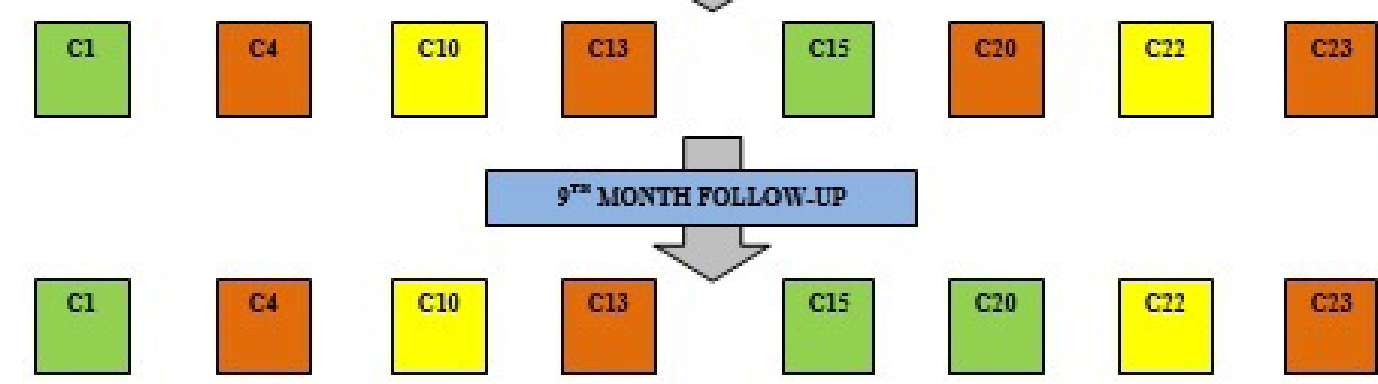

$9^{\text {Tz }}$ MONTH FOLLOW-UP
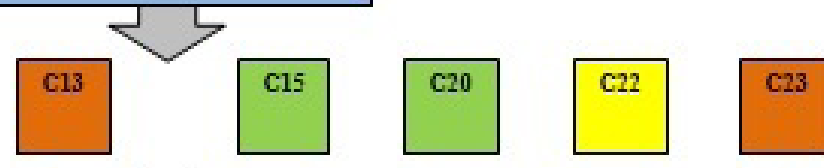

\section{YEAR FOLLOW-UP}
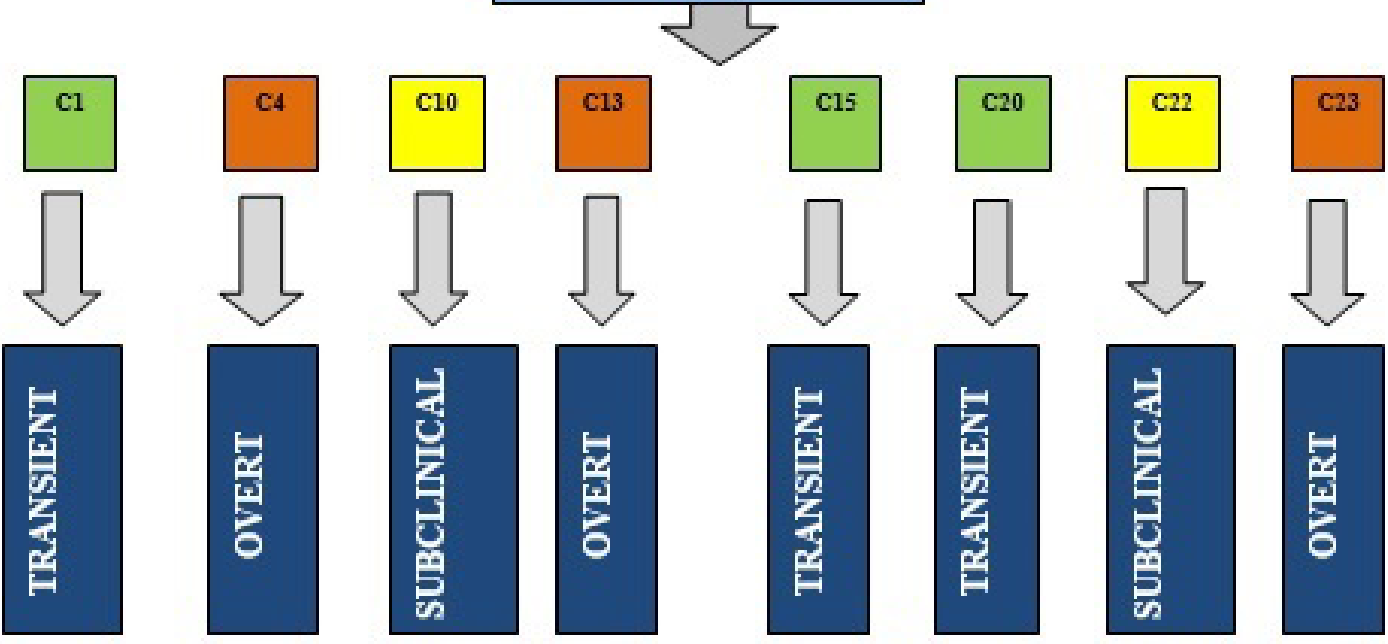

Fig.1. Results of progression of 25 patients in serial follow-up of 1 year 


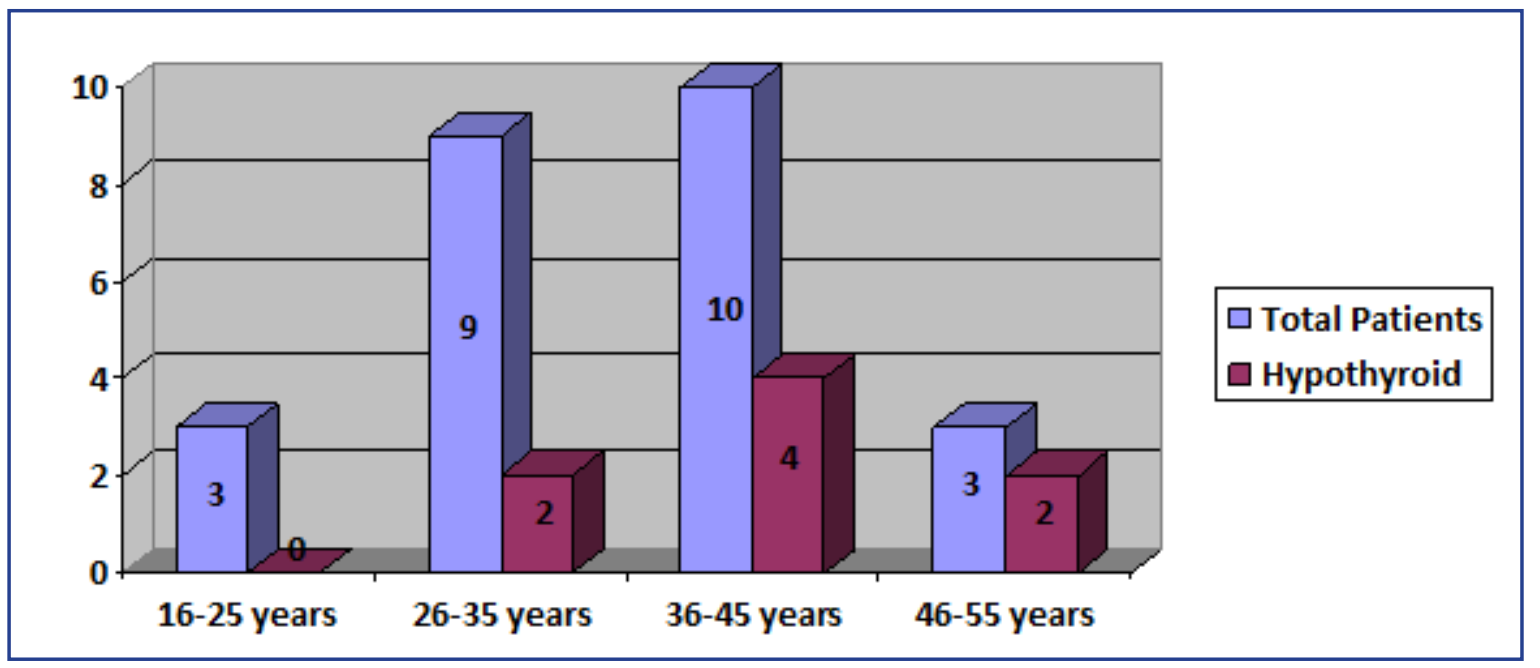

Fig.2. Distribution of patients according to age of presentation

suggests that chance of occurrence of post-operative hypothyroidism is directly proportional to the duration of thyroid swelling.

Histopathological report of each of the 25 patients, when correlated with the incidence of hypothyroidism (Fig. 5), showed that lymphocytic infiltration (4 out of 8 cases, $50 \%$ ) was most often associated with postoperative hypothyroidism, whereas when histopathology showed colloid goiter, the incidence was less (4 out of
14 cases, $28.6 \%)$.

It had also been contemplated that mostly the patients who underwent right-sided hemithyroidectomy were found to develop hypothyroidism (6 cases developed hypothyroidism out of total 13 patients who had underwent right-sided hemithyroidectomy).(Fig. 6)

Thus, from the above analysis, increased age of presentation, higher mean pre-operative serum TSH level, longer duration of thyroid swelling,

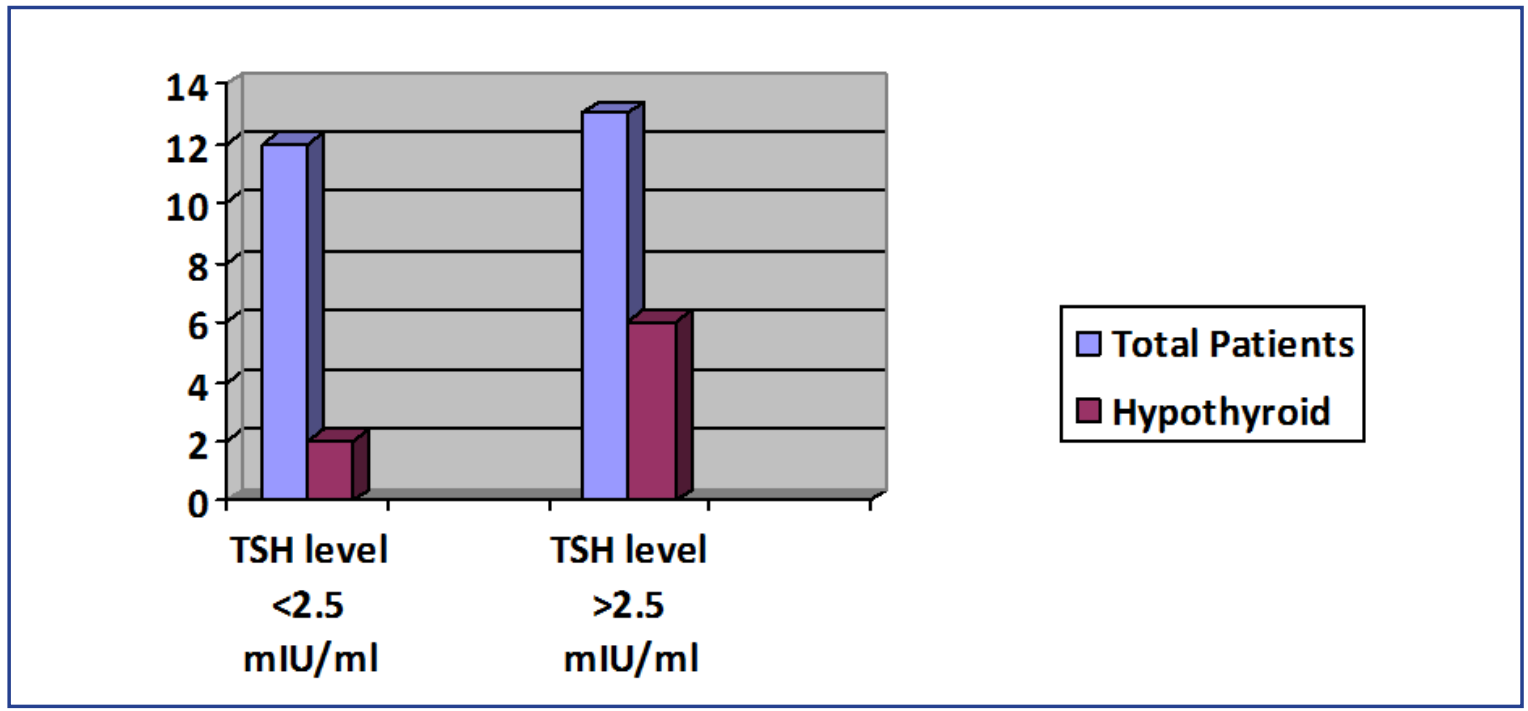

Fig.3. Distribution of patients according to pre-operative TSH level 


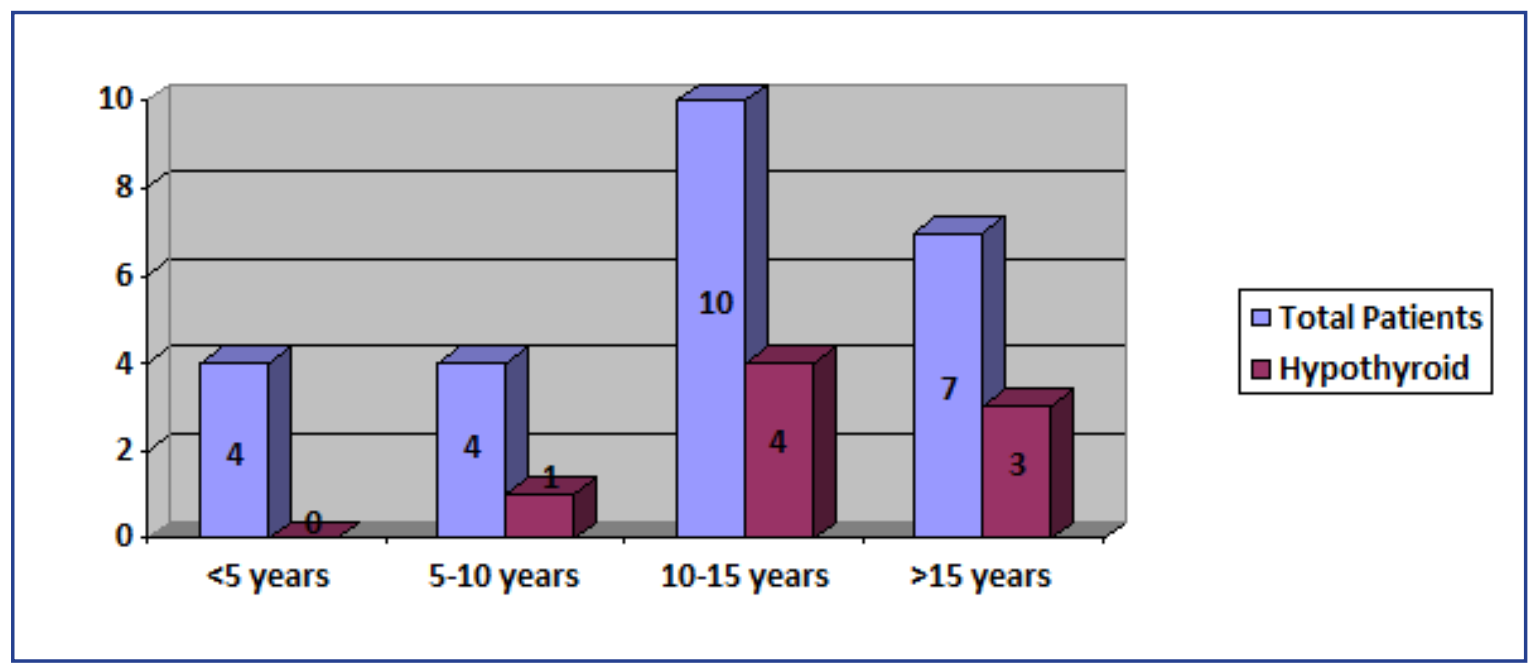

Fig.4. Distribution of patients according to the duration of thyroid swelling

lymphocytic infiltration in histopathology and rightsided hemithyroidectomy can be ascertained as the risk factors for post-operative hypothyroidism after hemithyroidectomy.

\section{Discussion}

Hypothyroidism, as a complication to total thyroidectomy, is not uncommon, but hypothyroidism after hemithyroidectomy is a topic of debate not only to otorhinolaryngologists, but also to general physicians and endocrinologists.

Certain literature mentioned that hypothyroidism is unusual after hemithyroidectomy. In reality, transient elevation of serum TSH is possible after hemithyroidectomy due to the remnant thyroid tissue activating the hypothalamo-pituitary-thyroid axis. ${ }^{7}$

Again, another school of thought was to put

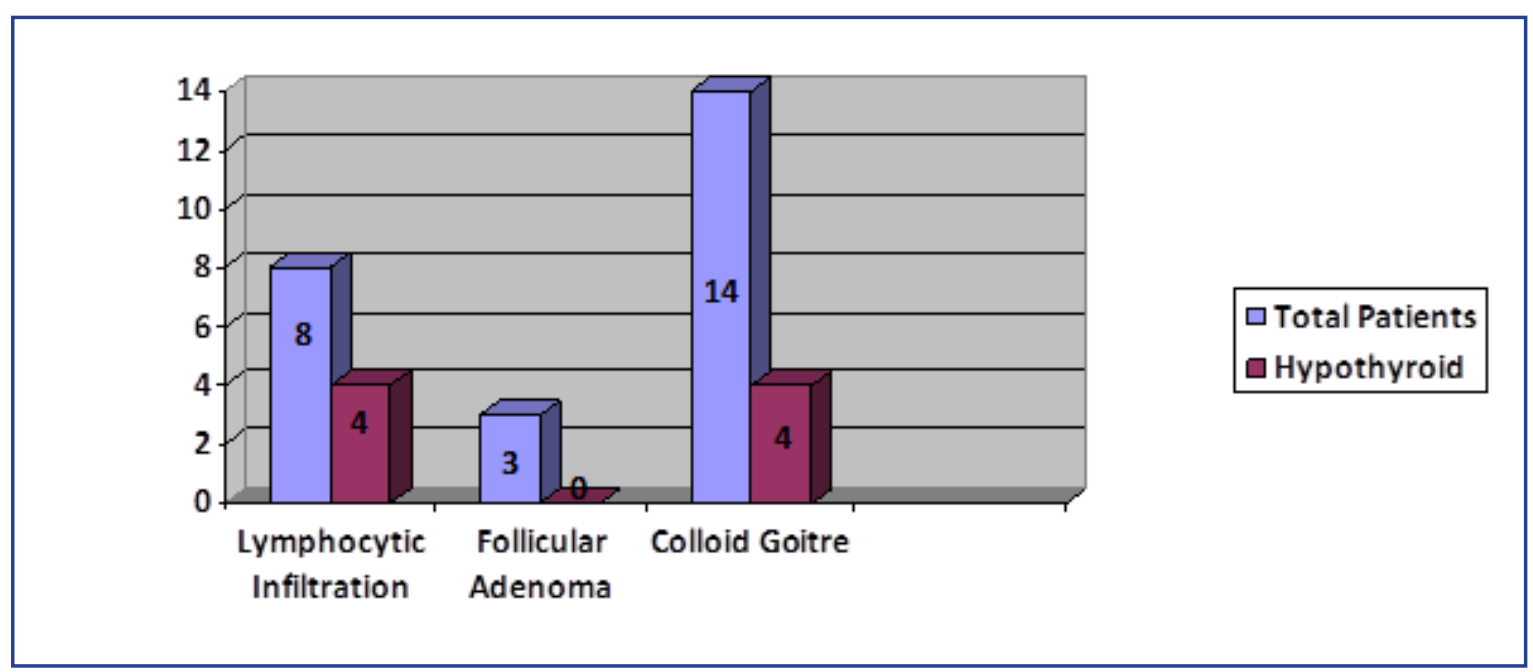

Fig.5. Distribution of patients according to histopathology 


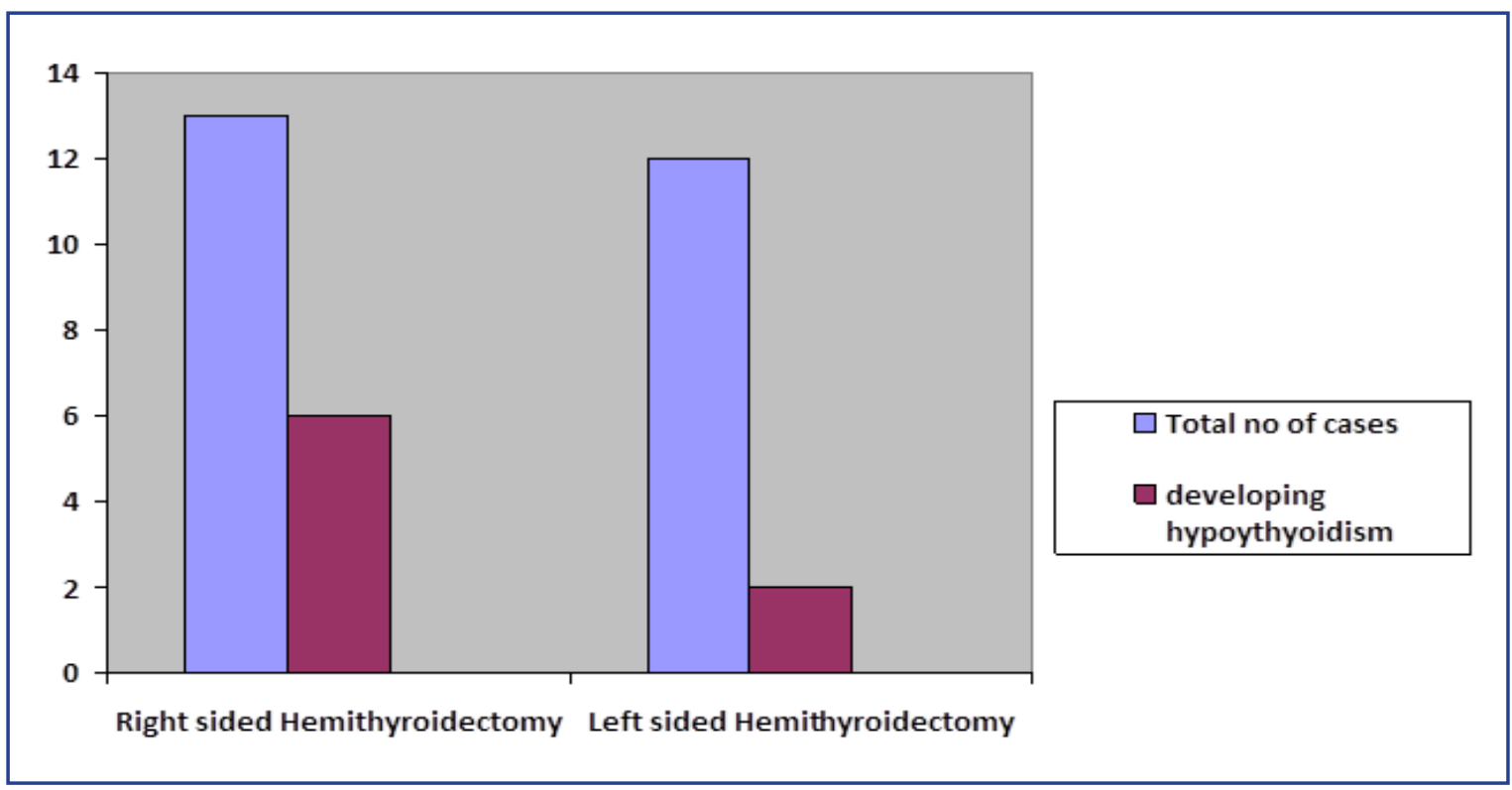

Fig.6. Distribution of patients according to the side of operation

the patients on regular levothyroxine suppression therapy in the immediate post-operative period after hemithyroidectomy, ${ }^{8}$ for preventing recurrent thyroid disease. But, it has been seen that levothyroxine therapy will mask the spontaneous recovery of thyroid function in the remaining lobe. ${ }^{9}$ Also, due to certain adverse effect of long term thyroid hormone replacement (osteoporosis, atrial fibrillation, etc. $)^{10}$ made the practice of regular levothyroxine supplementation obsolete.

Some of the recent researches have documented significant evidence of hypothyroidism after hemithyroidectomy and its association with certain risk factors. Most of the studies show incidence between $15-30 \% .^{1}$ In the present study, the incidence of postoperative hypothyroidism is $32 \%$ (20\% permanent, $12 \%$ transient). High normal value of pre-operative TSH is a very important predictor of post-operative hypothyroidism. ${ }^{11}$ According to Lankarani et al, increased age of presentation, along with prolonged duration of disease are important risk factors as they cause fibrosis of the gland. ${ }^{12}$ Side of operation, as per Ying et al and De Carlucci et al, is also a risk factor as because right lobe of thyroid is slightly larger than the left lobe. ${ }^{13,14}$ Wormald et al proved in their study that, lymphocytic infiltration in thyroid gland can cause postoperative hypothyroidism. ${ }^{15}$ Also there are certain other risk factors as evident in various literatures, like preoperative detection of thyroid auto-antibodies ${ }^{16}$ (antiTPO and anti-thyroglobulin antibody), body surface adjusted remnant thyroid volume, ${ }^{17}$ etc. But in the present study, those factors were not considered as this study is in a preliminary stage and can be considered later on. Early diagnosis is of much benefit by proper identification of the risk factors in the pre-operative period and stratification of the patients accordingly and subsequent meticulous follow-up in the risk group. Long term complications of prolonged hypothyroidism, cardiovascular and neuropsychiatric, such as pericardial effusion, left ventricular hypertrophy, Alzheimer's disease, myxoedema madness ${ }^{18}$ can be avoided if the patients are diagnosed in time.

The long term adverse effects are more dangerous in subclinical cases if not followed up properly. ${ }^{8}$ And therefore, the patients with subclinical hypothyroidism should be closely monitored for symptoms of hypothyroidism by serial follow up. As per various literature, routine thyroid hormone replacement is not recommended for cases of subclinical hypothyroidism with $\mathrm{TSH}<10 \mathrm{mIU} / \mathrm{L}$ and without signs/symptoms as in long term follow-up most of them returned to euthyroid state. ${ }^{19,20,21}$ In the present study also, there were cases of subclinical hypothyroidism who spontaneously 
became euthyroid without any particular intervention. We also got two cases of subclinical hypothyroidism, who continued to be subclinical till 1 year follow-up, and they are still under monitoring.

We should wait at least four to five half-lives of serum $\mathrm{TSH}(\mathrm{t} 1 / 2$ of serum TSH $=7$ days) before post-operative measurement of thyroid function ${ }^{22}$ as the thyroid hormone reserve maintains the euthyroid status. That is why, in the present study, the first measurement of serum TSH has been done at the end of the first month.

In the present study, there are few patients who had been detected to be hypothyroid in the early followup and progressively became euthyroid later on. So, the measurement of thyroid function only once at a relatively earlier stage without any further follow-up only diagnoses transient compensatory hypothyroidism and the true incidence remain unclear. ${ }^{1,8} \mathrm{~A}$ serial serum TSH measurement identifies those individuals who continue to remain biochemically hypothyroid and become symptomatic in long term and therefore require treatment with levothyroxine supplementation. The definite follow-up protocol is yet to be established, but the present study has successfully followed a protocol of monitoring the patients at 1st month, 3rd month, 6th month, 9th month and 1year post-operative period.

Although the present study has shown that hypothyroidism is not at all an unusual occurrence after hemithyroidectomy and there are certain risk factors, but as this is a preliminary report of an ongoing study, it will be too early to comment on the statistical significance. Longer duration of follow-up as well as inclusion of more parameters and further analysis will definitely strengthen the association between hypothyroidism and hemithyroidectomy and build a definite follow-up protocol. In the present scenario, awareness is called upon regarding this under-appreciated complication of hemithyroidectomy among all otorhinolaryngologists, general physicians and endocrinologists.

\section{Conclusion}

It is indicated from this preliminary report that there is a significant incidence of hypothyroidism occurring after hemithyroidectomy. The chance of developing post-operative hypothyroidism can be predicted from pre-operative assessment of certain risk factors, like, increased age of presentation, higher mean preoperative serum TSH level, longer duration of thyroid swelling, lymphocytic infiltration in histopathology and right-sided hemithyroidectomy. For diagnosis, there should be a serial thyroid function monitoring in every post-operative hemithyroidectomy patient. At present scenario, awareness is mostly needed regarding this under-appreciated complication of hemithyroidectomy.

\section{References}

1. Verloop H, Louwerens M, Schoones JW, Kievit J, Smit JWA, Dekkers OM. Risk of hypothyroidism following hemithyroidectomy: Systematic review and meta-analysis of prognostic studies. Journal of Clinical Endocrinology and Metabolism. 2012; 97(2):2243-55

2. Jameson JL, Weetman AP. Disorders of the thyroid gland (Chapter 341) in; Harrison's Principles of Internal Medicine 18th ed. Longo DL et al (Eds.). McGraw-Hill Companies. United state of America.2012; pp 2912-27

3. Ross DS. Serum thyroid-stimulating hormone measurement for assessment of thyroid function and disease. Endocrinol Metab Clin North Am. 2001; 30(2):245-64

4. Seiberling KA, Dutra JC, Bajarmovic S. Hypothyroidism following hemithyroidectomy for benign nontoxic thyroid disease. ENT- Ear, Nose and Throat Journal 2007; 86(5):295-9

5. Parle JV, Franlyn JA, Cross KW, Jones SC, Sheppard MC Prevalence and follow-up of abnormal thyrotrophin (TSH) concentrations in the elderly in the United Kingdom. Clin Endocrinol (Oxf). 1991; 34(1):77-83

6. Buchanan MA and Lee D. Thyroid auto-antibodies, lymphocytic infiltration and the development of post-operative hypothyroidism following hemithyroidectomy for non-toxic nodular goitre. Journal of the Royal College of Surgeons of Edinburgh 2001; 46(2): 86-90

7. Elsherbiny TM. Review: Post-thyroidectomy hypothyroidism. Egyptian Journal of Obesity, Diabetes and Endocrinology 2012; (2): 114-20

8. Lee J, Chung WY. Hypothyroidism After Hemithyroidectomy: Incidence, Risk Factors, Natural History and Management in, Hypothyroidism - Influences and Treatments, Springer D(Ed.), InTech, 2012;35-50. DOI: 10.5772/32153 http://cdn. intechopen.com/pdfs-wm/27823.pdf. accessed on 20/04/2016

9. Said M, Chiu V, Haigh PI. Hypothyroidism After Hemithyroidectomy. World Journal of Surgery 2013; 37: 283944

10. Klein I, Ojamaa K. Thyroid hormone and the cardiovascular system. N Eng J Med. 2001; 344(7): 501-9 
11. Miller FR, Paulson D, Prihoda TJ, Otto RA. Risk Factors for the Development of Hypothyroidism after Hemithyroidectomy. Arch Otolaryngol Head Neck Surg. 2006; 132: 36-8

12. Lankarani M, Mahmoodzadeh H, Poorpezeshk N, Soleimanpour B, Haghpanah V, Heshmat R. Hypothyroidism following thyroid surgery. Acta Medica Iranica 2008; 46(3): 225-32

13. Ying M, Yung DMC. Asymmetry of Thyroid Lobe Volume in Normal Chinese Subjects: Association with Handedness and Position of Esophagus. Anat Rec. 2009; 292 (2):169-74

14. De Carlucci Jr D, Tavares MR, Obara MT. Thyroid Function after Unilateral Total Lobectomy Risk Factors for Postoperative Hypothyroidism. Arch Otolaryngol Head Neck Surg. 2008; 134(10):1076-9

15. Wormald R, Sheahan P, Rowley S, Rizkalla H, Toner M, Timon C. Hemithyroidectomy for benign thyroid disease: who needs follow-up for hypothyroidism? Clin. Otolaryngol. 2008; 33:587-91

16. Koh YW, Lee SW, Choi EC, Lee JD, Mok JO, Kim HK, et al. Prediction of hypothyroidism after hemithyroidectomy: a biochemical and pathological analysis. Eur Arch
Otorhinolaryngol. 2008; 265:453-7

17. Moon H, Jung E, Park S, Jung TS, Jeong C, Ju Y. Thyrotropin Level and Thyroid Volume for Prediction of Hypothyroidism Following Hemithyroidectomy in an Asian Patient Cohort. World Journal of Surgery 2008; 32:2503-8

18. Wartofsky L, van Nostrand, D. Overt and subclinical hypothyroidism in women Obstet Gynecol Surv. 2006; 61(8):535-42

19. Col NF, Surks MI, Daniels GH. Subclinical thyroid disease: clinical applications. JAMA 2004; 291(2):239-243

20. Surks MI, Oritz E, Daniels GH, Sawin CT, Col NF, Cobin RH,Franklin JA, Hershman JM, Burman KD, Denke MA, Gorman C, Cooper RS, Weissman NJ. Subclinical thyroid disease: scientific review and guidelines for diagnosis and management. JAMA 2004; 291(2):228-38

21. Cooper DS, Biondi B. Subclinical thyroid disease. Lancet 2012; 379(9821):1142-56

22. Benediktsson R, Toft AD. Management of the unexpected results: Compensated hypothyroidism. Postgrad Med J. 1998; $74: 729-32$ 